\title{
Optimized depth-resolved estimation to measure optical attenuation coefficients from optical coherence tomography and its application in cerebral damage determination
}

\author{
Jian Liu \\ Ning Ding \\ Yao Yu \\ Xincheng Yuan \\ Shuzhuo Luo \\ Jingmin Luan \\ Yuqian Zhao \\ Yi Wang \\ Zhenhe Ma
}




\title{
Optimized depth-resolved estimation to measure optical attenuation coefficients from optical coherence tomography and its application in cerebral damage determination
}

\author{
Jian Liu, ${ }^{a}$ Ning Ding, ${ }^{b}$ Yao Yu, ${ }^{c}$ Xincheng Yuan, ${ }^{d}$ Shuzhuo Luo, ${ }^{b}$ Jingmin Luan, ${ }^{c}$ Yuqian Zhao, ${ }^{\text {a }}$ Yi Wang, ${ }^{a}$ and \\ Zhenhe $\mathrm{Ma}^{\mathrm{a}, \star}$ \\ aNortheastern University at Qinhuangdao, School of Control Engineering, Qinhuangdao, China \\ ${ }^{b}$ Northeastern University, School of Sino-Dutch Biomedical and Information Engineering, Shenyang, China \\ 'Northeastern University at Qinhuangdao, School of Computer and Communication Engineering, Qinhuangdao, China \\ dUniversity of Michigan, School of Biomedical Engineering, Ann Arbor, Michigan, United States
}

\begin{abstract}
The optical attenuation coefficient (OAC) reflects the optical properties of various tissues or tissues of the same type under different physiological conditions. Quantitative measurement of OAC from optical coherence tomography (OCT) signals can provide additional information and can increase the potential for OCT applications. We present an optimized depth-resolved estimation (ODRE) method that derives a precise mapping between the measured OCT signal and the OAC. In contrast to previous depth-resolved estimation (DRE) methods, the optimized method can estimate the OAC in any depth range and ignore whether the light is completely attenuated. Numerical simulations and phantom experiments are used to verify its validity, and this method is applied to detect cerebral damage. In combination with OCT angiography, real-time observation of the change of blood perfusion and the degree of cerebral damage in mice with focal cerebral ischemia provides important information to help us understand the temporal relationship between brain damage and ischemia. (c) The Authors. Published by SPIE under a Creative Commons Attribution 4.0 Unported License. Distribution or reproduction of this work in whole or in part requires full attribution of the original publication, including its DOI. [DOI: 10.1117/1.JBO.24.3.035002]
\end{abstract}

Keywords: optical coherence tomography; optical attenuation coefficient; optimized depth-resolved estimation; cerebral ischemia. Paper 180524RR received Aug. 31, 2018; accepted for publication Feb. 14, 2019; published online Mar. 4, 2019.

\section{Introduction}

Optical coherence tomography (OCT) is a promising noninvasive imaging modality in vivo for label-free three-dimensional tissue imaging in vivo. ${ }^{1}$ In recent years, methods for calculating attenuation coefficients from OCT signals to identify various tissue types and obtain their pathological status information have begun to attract the attention of scholars. This idea first came from ultrasonic imaging. ${ }^{2}$ When the ultrasound or infrared light penetrate the tissue, the intensity decays exponentially, and therefore the attenuation coefficient reflects the acoustic/ optical characteristics of different tissues or the same type of tissues under different physiological conditions. According to some basic research, due to the lack of oxygen and energy, cerebral ischemia is accompanied by changes in cellular morphology, which optical attenuation coefficient (OAC) is sensitive to. Consequently, OAC increases during cerebral ischemia. ${ }^{3}$ Observations of ischemia tissue by transmission electron microscopy suggest that the increase in OAC is due to deformation or enlargement of mitochondria and dendrites expansion. ${ }^{4}$ Through quantitative measurement of OAC, cerebral tissue damaged by ischemia can be effectively labeled and evaluated. This method provides additional information and increases the potential of OCT in exploring cerebral infarction.

The calculation of OAC from OCT signals is usually performed by fitting an exponential curve through OCT depth

*Address all correspondence to Zhenhe Ma, E-mail: mazhenhe@163.Com profiles. Nevertheless, this method requires precise layering, and the OACs of different layers are obtained separately. 5,6 Because the curve-fitting method needs to be performed within a certain depth range, the image should only contain one tissue type, or the region of interest (ROI) must be selected before fitting the curve. However, the selection of ROI may have a negative impact on the fitting results, so this method may be very limited in practical applications. Some other improvements have also been proposed, including, for example, the axial point spread function (PSF), ${ }^{7}$ but none so far has fundamentally dealt with the limitations of the curve-fitting approach.

Recently, a depth-resolved estimation (DRE) method has been developed to convert each pixel of an OCT signal to a corresponding $\mathrm{OAC}$ value. ${ }^{8}$ This method estimates $\mathrm{OAC}$ with much higher resolution by modeling OCT signals based on single scattering by tissue and provides per-pixel OAC values without prior layering. However, increasing errors are observed near the end of the imaging depth range. In addition, it does not take the OCT noise floor into account, which may affect the accuracy of the OAC estimation. Baran et al. ${ }^{9,10}$ combine the DRE method with the en face sorted average intensity projection (sAIP) method and calculate the average $\mathrm{OAC}$ for a specific en face location. They observe changes in the OAC of cerebral cortex during cerebral ischemia-reperfusion in mice and find that, during occlusion, the average OAC in the ischemic region is greater than the baseline condition. After reperfusion, the average OAC at the middle cerebral artery (MCA) side still keeps increasing significantly despite partial blood flow recovery. ${ }^{9,10}$ This study preliminarily demonstrates the potential of OCT in determining 
the area of cerebral infarction. But the algorithm itself has not been improved.

In this paper, we propose an optimized depth-resolved estimation (ODRE) method to reduce the errors when estimating OAC. Numerical simulations and phantom experiments are used to verify its validity. We also apply this method to define the degree of cerebral damage. The changes in blood perfusion and the degree of brain injury in mice with focal cerebral ischemia are observed in real time, and the relationship between the two is analyzed.

\section{Optical Coherence Tomography System and Signal Processing}

In this study, a spectral-domain OCT (SD-OCT) system was employed, which is similar to our previous work. ${ }^{11,12}$ Briefly, a superluminescent diode with a central wavelength of $1310 \mathrm{~nm}$ and a bandwidth of $65 \mathrm{~nm}$ was used as a light source, providing an $\sim 8.9-\mu \mathrm{m}$ axial resolution in the tissue. In the sample arm, an achromatic lens with 30 -mm focal length was used to achieve theoretical lateral resolution of $\sim 9 \mu \mathrm{m}$. The data were acquired with 125 frames per second, where each B-frame datum was composed of 400 A-lines covering $\sim 1.5$-mm total distance. The slow axis (C-scan), consisting of $2000 \mathrm{~B}$-frames, covers the same distance by repeatedly scanning five times at each position (a total of 400 locations). In SD-OCT, the presence of a fixedpattern noise results in strong erroneous horizontal lines on the image, which usually comes from the non-flatness of the reference spectrum that is to be interfered with the signal light. It may also be produced by optical interferences that occur in the middle of the optical pathway of the reference light. It can be removed by generating a reference spectrum through recording the reference arm spectrum or averaging many spectra of an actual measurement. ${ }^{13}$ However, recording a reference spectrum is not always convenient and effective, ${ }^{14}$ so we used the latter. Each interferometric spectrum is subtracted by the mean spectrum to remove the static component. This method is popular for SD-OCT, due to its simple practical implementation and inherent immunity to reference changes.

OCT signal intensity is affected by the focus location. Therefore, the confocal properties of the OCT system must be taken into account when extracting OAC quantitatively. Recently, Faber et al. derived the general expression of the confocal axial PSF, ${ }^{15,16}$ which is described as

$I(z) \propto h(z) \cdot e^{-2 \mu z}$,

$h(z)=\left[\left(\frac{z-z_{c f}}{z_{R}}\right)^{2}+1\right]^{-1}$,

where $\mu$ is the OAC and $z$ is the signal depth. The function $h(z)$ is the axial PSF, $z_{\mathrm{cf}}$ is the position of the confocal gate, and $z_{R}$ is the "apparent" Rayleigh length used to characterize the axial PSF:

$z_{R}=\alpha \pi n w_{0}^{2} / \lambda$

where $w_{0}$ is the minimum beam radius, $\lambda$ is the center wavelength of the light source, $n$ is the refractive index (we used $n=1.353$ ), and $\alpha$ is used to distinguish specular reflection $(\alpha=1)$ from diffuse reflection $(\alpha=2)$. In this paper, $z_{R} \approx 129 \mu \mathrm{m}$. The bias could be removed by dividing it by axial PSF $h(z) .{ }^{17}$ To visualize the volumetric microvasculature down to the capillary level, an OCT angiography technique was utilized.

\section{Optical Attenuation Coefficient Estimation}

\subsection{Depth-Resolved Estimation}

Vermeer et al. ${ }^{8}$ introduce the model as shown in Eq. (4), where $I[z]$ is the OCT signal of a pixel and $\mu[z]$ is the OAC, both at depth $z$, and $\Delta$ is the pixel size (usually related to the axial resolution of the OCT system):

$\mu[z]=\frac{I[z]}{2 \Delta \sum_{i=z+1}^{\infty} I[i]}$.

By using this equation, the mapping relationship between the OCT signal intensity and the OAC is established. For the DRE method, the number of pixels available for calculation is limited due to the limited detection depth of the OCT system. Therefore, the OAC should be approximated as

$\mu[z] \approx \frac{I[z]}{2 \Delta \sum_{i=z+1}^{N} I[i]}$,

where $N$ is the number of the pixels within a limited depth. The one problem with DRE is that the approximate processing of OAC is impractical. In Vermeer et al.'s model, the boundary condition is $I(\infty)=0$, which means that the detection depth is infinite. However, in the actual environment, it is apparent that infinite depth is impossible. In this case, the DRE model uses a finite depth $N$ instead of $\infty$, assuming that the light decays completely in a limited range of penetration. For small depths, it means that $z$ is small enough, $\sum_{i=z+1}^{N} I[i] \approx \sum_{i=z+1}^{\infty} I[i]$ can be considered satisfactory. As $z$ increases, the difference between $z$ and $N$ decreases, resulting in an increase in the difference between $\sum_{i=z+1}^{N} I[i]$ and $\sum_{i=z+1}^{\infty} I[i]$, which is regarded to be an error. When $z=N-1$, the error peaks.

Another problem is that the DRE method has not taken the OCT noise floor into account. The noise floor includes electrical noise of the photodetector, shot noise, and relative intensity noise from the reference arm light. ${ }^{18}$ If the OCT signal is fully attenuated when it reaches the depth $z_{S}$, then all of the depths from $z_{S}+1$ to $N$ may be noise. The DRE method adds a large amount of noise to the denominator of Eq. (5), and the resulting OAC may be smaller than it should be. In this paper, we propose the ODRE method to solve these problems.

\subsection{Optimized Depth-Resolved Estimation}

The DRE method is suitable for the boundary condition $I(\infty)=0$, so Eq. (4) can be used for subsequent calculations and the OAC for the last point $N$ should be expressed as

$\mu[N]=\frac{I[N]}{2 \Delta \sum_{i=N+1}^{\infty} I[i]}$,

where the equation can be rewritten as

$2 \Delta \sum_{i=N+1}^{\infty} I[i]=\frac{I[N]}{\mu[N]}$.

With this equation, we can supplement the data after the last point $N$, because $\sum_{i=z+1}^{\infty} I[i]$ can be split into $\sum_{i=z+1}^{N} I[i]$ and $\sum_{i=N+1}^{\infty} I[i]$. Equation (4) can be rewritten as 


$$
\begin{aligned}
\mu[z] & =\frac{I[z]}{2 \Delta \sum_{i=z+1}^{N} I[i]+2 \Delta \sum_{i=N+1}^{\infty} I[i]} \\
& =\frac{I[z]}{2 \Delta \sum_{i=z+1}^{N} I[i]+\frac{I[N]}{\mu[N]}},
\end{aligned}
$$

where $\mu[N]$ in Eq. (8) is unknown. To determine it, a piece of data was intercepted from the ranging depth of the system and then fitted with an exponential curve (as shown in Fig. 3). The fitting model is $y=a \cdot \exp (-2 \mu z)+b$. The fitted result $\mu$ is the average attenuation coefficient of this piece of data and can be considered as the best approximation of $\mu[N]$. The following is a detailed description of each step of the algorithm:

1) Surface location: The surface position was determined independently for each A-scan by using the moving summation window method proposed in literature. This method detects the first peak intensity of the summation of a moving window, and the highest peak of the moving window array would be the top surface pixel of the cortex.

2) Signal cropping: After surface location, we select a specific depth range within the sample for data analysis. To avoid the effect of the noise floor on the OAC, the OCT signal in this depth range should generally have a signal-to-noise ratio (SNR) of $5 \mathrm{~dB}$ higher than the noise floor. ${ }^{19}$ Figure 1 shows an example of OCT signal cropping. The black curve is a typical average OCT signal of a mouse cerebral cortex. The blue curve indicates the noise floor measured with the reference light only. The red curve represents the position that is $5 \mathrm{~dB}$ above the noise floor. The small black square is where the signal is cropped.

3) Fitting length determination: Before fitting the end-ofline data of the cropped OCT signal, we also need to determine the fitting length. To find the best pixel number required to produce a reasonable curve fitting result, signals with different lengths at the bottom layer are tested. The fitting results are shown in Fig. 2.

As can be seen from Fig. 2, with the increase in the number of pixels, the standard deviation of the obtained OAC gradually decreases, thus indicating that the more

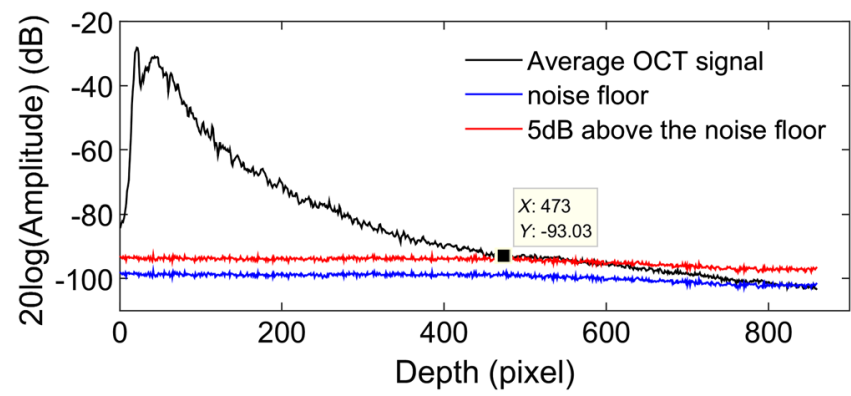

Fig. 1 OCT signal cropping. The black curve is a typical average OCT signal. The blue curve indicates noise floor measured with the reference light only. The red curve represents the position that is $5 \mathrm{~dB}$ above the noise floor. The small black square is where the signal is cropped. All the curves were obtained by averaging 100 consecutive measurements to facilitate comparison.

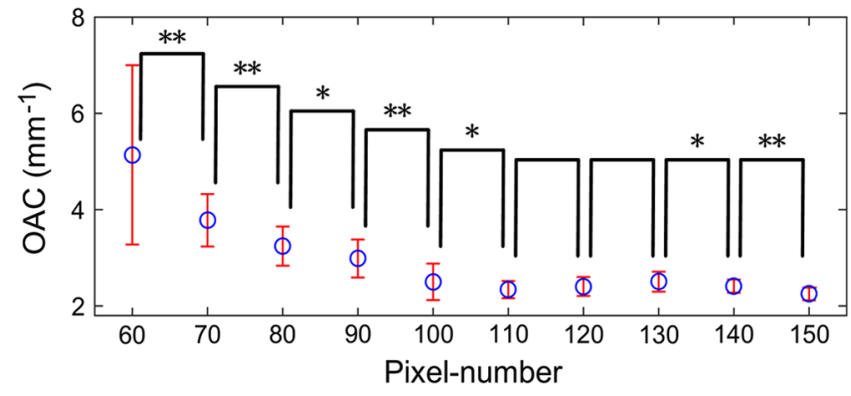

Fig. 2 The relationship between the pixel number and the OAC. All values reported as the mean $\pm \mathrm{S}$. E. M; $\left(^{*}\right)$ represents $p<0.05$ and $\left({ }^{* *}\right)$ represents $p<0.01$.

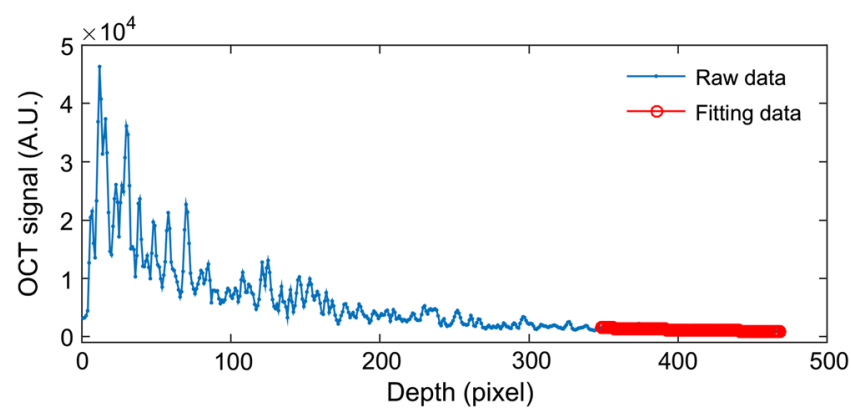

Fig. 3 Exponential fitting is performed for the end of the imaging depth (120 pixels). The solid blue line is a typical averaged A-scan OCT signal. The solid red line represents the result of the fitting.

pixels there are, the more stable the result will be. On the other hand, the more pixels, the greater is the probability that these pixels will come from different types of tissue. We performed a $t$-test on adjacent results. It was found that there was no significant difference in the fitting results when the number of pixels was 110,120 , and 130. Therefore, without the loss of generality, we use 120 as the fitting length. Note that, the number of pixels used in different samples may be different.

4) Exponential fitting: As OCT signals are usually very noisy, we averaged five adjacent A-scans when performing exponential fitting to increase the SNR. The nonlinear fitting function "nlinfit" in MATLAB is used to automatically fit the fitting process after the fitting length is determined. The fitting model is $y=$ $a \cdot \exp (-2 \mu z)+b$. The initial value of $a, \mu$, and $b$ is defined as $[1000,0.1,100]$. Figure 3 is the fitting process. After fitting, we make $\mu[N]=\mu$ and $I[N]=y[N]$. Finally, we bring $\mu[N]$ and $I[N]$ into Eq. (8). The OACs were estimated A-scan by A-scan.

\section{Results}

\subsection{Numerical Simulations}

Reliable testing and simulation play an important role in the innovation of optical imaging, measurement techniques, and data analysis algorithms. To evaluate the effectiveness of the ODRE method experimentally, a series of numerical simulations were performed with and without noise. We first simulated a beam of light propagating through a homogeneous medium 

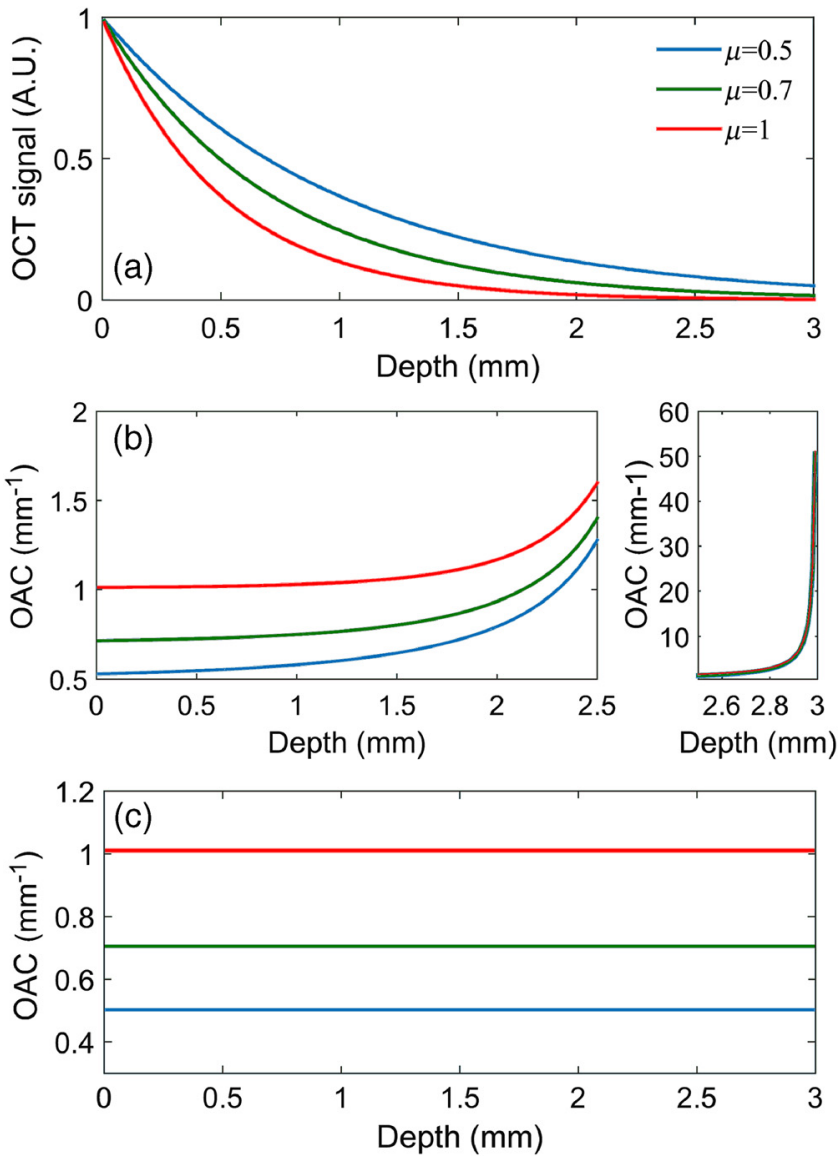

Fig. 4 Numerical simulation without noise. (a) Simulated OCT signals. (b) DRE results; the small graph to the right of (b) shows the depth from 2.5 to $3 \mathrm{~mm}$, indicating a huge error. (c) ODRE results.

without noise and using three sets of OACs $(0.5,0.7,1)$. The observed depth was $3 \mathrm{~mm}$, and the pixel size $\Delta$ was $0.01 \mathrm{~mm}$. As shown in Fig. 4(a), for ease of calculation, we assumed that the initial light intensity is 1 . The OACs of the simulated data were estimated by the method of DRE and ODRE, respectively. For ODRE method, the number of pixels used for exponential fitting is $100(1 \mathrm{~mm})$. The results are shown in Figs. 4(b) and $4(\mathrm{c})$.

Generally, the OACs at different depths in a homogeneous medium should be the same, but there is increasing error in the results of DRE and its maximum is $\mu[N-1]=I[N-1] /$ $2 \Delta I[N] \approx \frac{1}{2 \Delta}=50 \mathrm{~mm}^{-1}$. It should be noted that the estimation error of the DRE method exists at any depth and not just at the end of the data. On the other hand, OACs obtained by the ODRE method are satisfactory and consistent with the preset OACs $(0.5,0.7$, and 1$)$.

As mentioned above, DRE method can only be used if almost all light is attenuated within the recorded imaging depth range. In practice, the OCT data does not necessarily satisfy such a depth range. As a result, an unacceptable error is generated, when the DRE method is used. Here, we selected three depth ranges of $0.5,1$, and $1.5 \mathrm{~mm}$, respectively. The shallower the depth, the more light is left [as shown in Fig. 5(a)]. The OACs were estimated within these limited depth ranges by DRE and ODRE. The results are shown in Figs. 5(b) and 5(c), respectively.

As shown in Fig. 5, a major error can occur if the optical signal attenuation is not completed within the limited depth
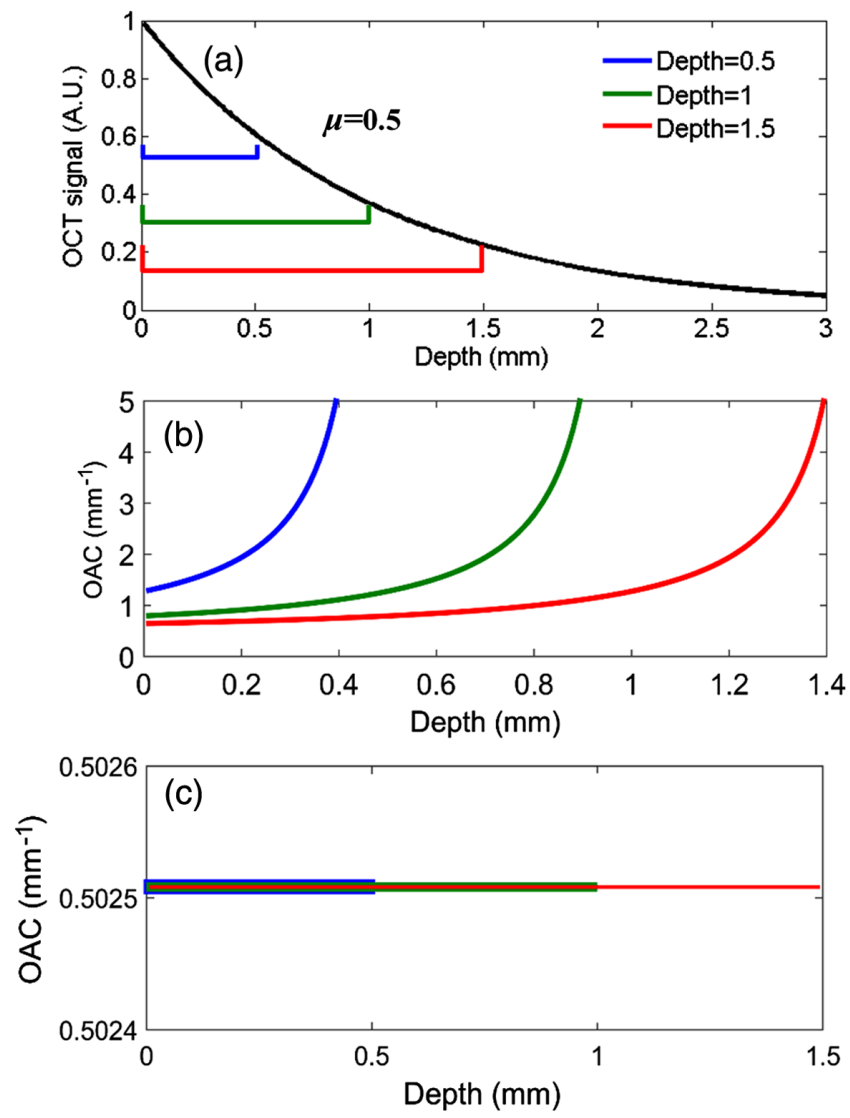

Fig. 5 Numerical simulation for homogeneous media with finite depth (a) simulated OCT signals, the preset OAC is $0.5 \mathrm{~mm}^{-1}$, (b) DRE results, and (c) ODRE results (the error is only $0.5 \%$ ).

range. The smaller the depth range, the sooner the error occurs. Instead, the ODRE method can be used to estimate the OACs in any depth range with negligible error.

OCT images are often affected by speckle noise, which is a multiplicative noise of the coherent imaging system. ${ }^{20,21}$ To accurately reflect the performance of the proposed method in OAC estimation in a noisy environment, three sets of numerical signals were set up, and each set was actually a combination of multiplicative noises and the corresponding analog datasets used in Fig. 4. The increasing process of the noise follows the development:

$I^{\prime}(z)=I(z) \times(1+\varepsilon)$.

Here, $I(z)$ is the analog signal at depth $z$, with its counterpart used in Fig. 4. $I^{\prime}(z)$ is the signal with multiplicative noise added in and $\varepsilon$ is a uniformly distributed random noise with a specific amplitude. The amplitude distribution of speckles in an OCT signal can be expressed as the ratio of the standard deviation to the average value of the signal. ${ }^{22}$ According to Pircher's theory, the contrast of the speckle pattern is $0.52 .^{23}$ Speckle noise can be offset by multiple averages. In this paper, we repeat the acquisition five times at each position, and the speckle noise is reduced to $1 / \sqrt{5}$ of the original. Thus, the actual contrast of the speckle pattern is 0.23 . So, we next simulated a signal with a multiplicative noise of $23 \%$ amplitude, as shown in Fig. 6 .

Figure 6(a) shows the original simulated data, and Figs. 6(b) and $6(\mathrm{c})$ show the estimated value of the OACs obtained by the DRE and the ODRE, respectively. 

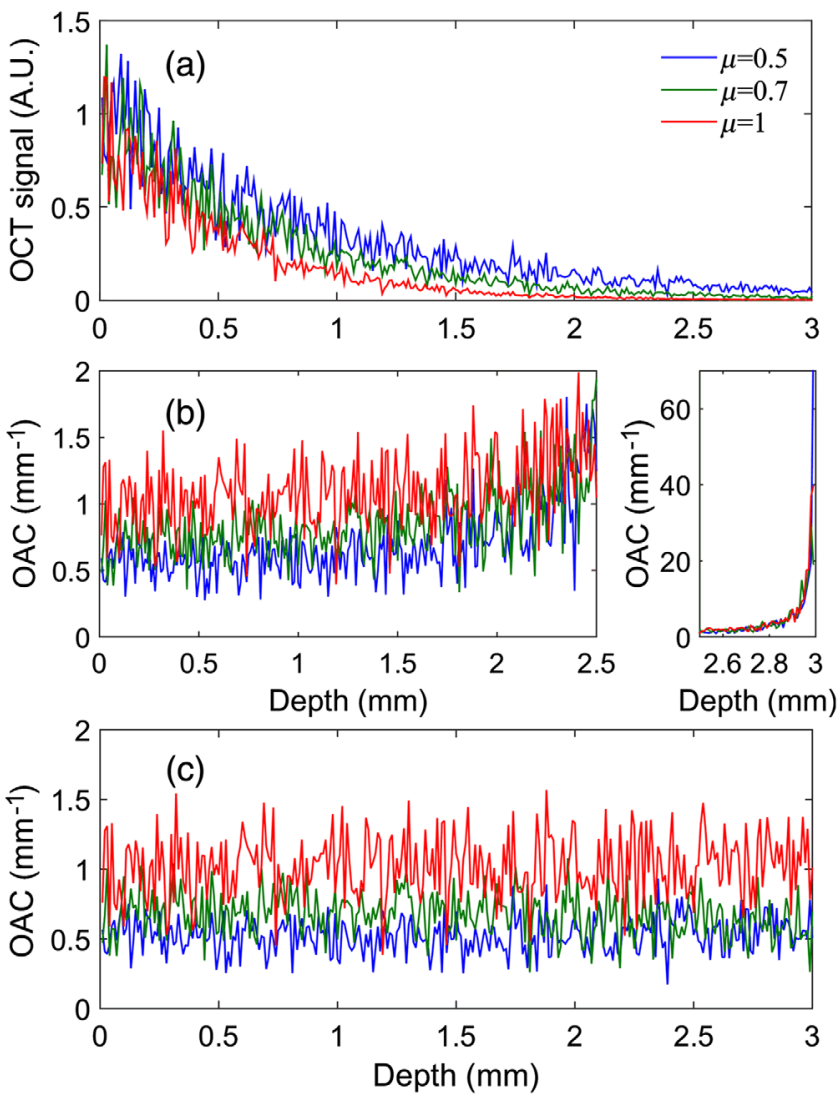

Fig. 6 Numerical simulation with random noise. (a) Simulated OCT signals. (b) DRE results; the small graph to the right of (b) shows the depth from 2.5 to $3 \mathrm{~mm}$. (c) ODRE results.
According to Fig. 6, this series of results are similar to that of Fig. 4. Again, the results of ODRE are still consistent with the preset OACs, but the difference is that the former fluctuates by noise. These convincing simulation results show that the proposed method can accurately estimate the OACs in a random depth regardless of the noise.

\subsection{Phantom Experimental Results}

Four liquid optical phantoms comprising different Intralipid concentrations were prepared. The volume-to-volume (v/v) ratios used were $\sim 5 \%, 10 \%, 15 \%$, and $20 \%$ (diluted in distilled water). Three methods (exponential fitting, DRE, and ODRE) were used to estimate the OAC. For the DRE method, the entire imaging depth was used for OAC estimation. For the ODRE method, only $0.5-\mathrm{mm}$ depth range was used. The number of pixels used for exponential fitting is $60(0.15 \mathrm{~mm})$. Figure 7 shows the experimental results of $20 \%$ phantom. Figure 7(a) shows the OCT intensity image, Fig. 7(b) shows the corresponding OAC image obtained by DRE using Eq. (4), and Fig. 7(c) shows the corresponding OAC image obtained by ODRE using Eq. (8).

Since the phantoms are homogeneous, the exponential fitting method can be applied to the entire depth range, and the results are credible and can be used as a standard for evaluating the other methods. ${ }^{8}$ The blue solid line in Fig. 7(d) is shown as the optical signal data for the leftmost column (A-scan) of Fig. 7(a). The exponential fitting method is used to process the data, and the result is shown in the red solid line in Fig. 7(d). The result $(\mu)$ is $\sim 3.0 \mathrm{~mm}^{-1}$. Figure $7(\mathrm{e})$ is the results of ODRE and DRE. It can be seen that the OAC curve within the depth range obtained by the ODRE method is even, and the average OAC value (red dotted line) is consistent with the exponential fitting result.
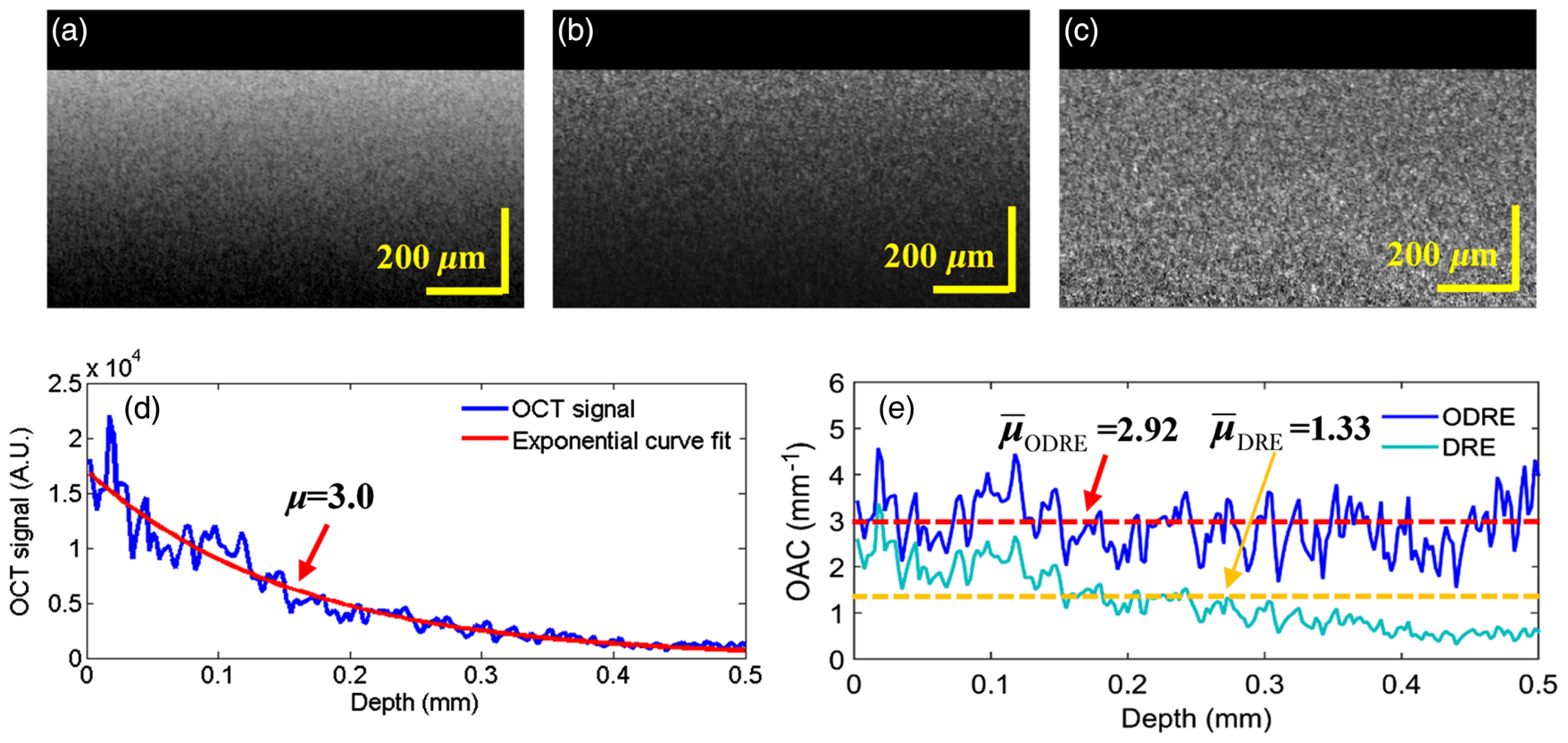

Fig. 7 (a) OCT intensity image from uniform phantoms; (b) and (c) corresponding OAC image obtained by DRE and ODRE, $\Delta \approx 0.0025 \mathrm{~mm}$. (d) A typical OCT A-scan within (a) and the result of exponential curve fitting. The result $(\mu)$ is $\sim 3.0 \mathrm{~mm}^{-1}$. (e) The blue curve is the estimated result obtained by the ODRE method. The red dotted line is the corresponding average OAC at the depth range of $0.5 \mathrm{~mm}$, which is consistent with the exponential fitting result. The cyan curve is the estimated result obtained by the DRE method; the OAC value is underestimated due to the accumulation of excessive noise during the estimation process. The orange dotted line is the corresponding average OAC, which is significantly smaller than the result of exponential fitting. 
Table 1 OAC estimated by the exponential fitting, DRE, and ODRE methods from the uniform phantoms.

\begin{tabular}{lccc}
$\begin{array}{l}\text { Concentration } \\
(\%)\end{array}$ & $\begin{array}{c}\text { Exponential } \\
\text { fitting }\left(\mathrm{mm}^{-1}\right)\end{array}$ & $\begin{array}{c}\text { DRE } \\
\left(\mathrm{mm}^{-1}\right)\end{array}$ & $\begin{array}{c}\text { ODRE } \\
\left(\mathrm{mm}^{-1}\right)\end{array}$ \\
\hline 5 & $1.18 \pm 0.12$ & $0.93 \pm 0.16$ & $1.16 \pm 0.21$ \\
10 & $1.49 \pm 0.08$ & $1.03 \pm 0.18$ & $1.40 \pm 0.26$ \\
15 & $2.12 \pm 0.15$ & $1.20 \pm 0.18$ & $2.05 \pm 0.29$ \\
20 & $3.0 \pm 0.17$ & $1.36 \pm 0.20$ & $2.92 \pm 0.22$ \\
\hline
\end{tabular}

The OAC value obtained by the DRE method is underestimated due to the accumulation of excessive noise during the estimation process. The OAC of each phantom obtained from the exponential fitting, DRE, and ODRE are listed in Table 1.

It can be seen from Table 1 that the OAC obtained by the DRE method is generally small, and the error is larger with the increase in the concentration. This is because the faster the signal attenuate, the greater is the noise accumulated by the DRE method on the denominator of Eq. (5) and the greater is the error.

To better validate the capabilities of ODRE in practical applications, a multilayer phantom was created. The phantom consisted of three thin layers $(15 \%, 5 \%$, and $10 \%), 0.25 \mathrm{~mm}$ per layer. Each layer is separated by a $0.12-\mathrm{mm}$ coverslip. The multilayer phantom has a total depth of $1.2 \mathrm{~mm}$. The number of pixels used for exponential fitting is $60(0.15 \mathrm{~mm})$.

The experimental results are shown in Fig. 7.

Figure 8(a) shows the OCT intensity image of the multilayer phantom, A E represent different layers. Among them, A, C, and $\mathrm{E}$ are $15 \%, 5 \%$, and $10 \%$ of phantom, respectively. Here,
B and D are the coverslips. Figure 8(b) shows the corresponding OAC image obtained by ODRE. Figure 8(c) is a typical average A-scan within Fig 8(a), Fig. 8(d) is the OAC corresponding to Fig. 8(c). The OAC results for the three-layer phantom are $\mu(A)=2.04 \pm 0.20, \mu(C)=0.83 \pm 0.26$, and $\mu(E)=1.20 \pm$ 0.25 , respectively. A $T$-test is performed between the OACs obtained by the ODRE from multilayered samples and the OAC obtained by the exponential curve-fitting from homogenous sample. There is no significant difference for layer $\mathrm{A}$ (15\%), and there is a significant difference for layer $\mathrm{C}(5 \%)$ and E $(10 \%), p<0.05$. This may be due to a lower SNR at a deeper depth.

\subsection{In Vivo Mouse Brain Imaging}

About 3-month-old C57/616 mice (20 to $30 \mathrm{~g}$ ) were used in the study. All procedures were performed in accordance with the Animal Ethics and Administrative Council of Northeastern University. All efforts were made to minimize animal suffering and to reduce the number of animals used. Surgical anesthesia was induced with sodium pentobarbital (3\%, $5 \mathrm{mg} / 100 \mathrm{~g}$, IP). The scalp was cut along the midline of the skull, and the interparietal bone was exposed by pulling the skin to the side of the head. A standard $3 \times 3 \mathrm{~mm}^{2}$ cranial window, covering the distal branches of MCA and the anterior cerebral artery (ACA), was created in the left parietal cortex, $0.5-\mathrm{mm}$ lateral from the sagittal suture and $0.5-\mathrm{mm}$ posterior from the bregma. A circular coverslip was placed on the exposed brain surface and sealed on bone with a dental cement. ${ }^{24}$ After surgery, the mice were placed into the sample arm of the OCT system and prepared for baseline data acquisition.

The model of the focal cerebral ischemia used in our experiments was based on the previously described endothelin-1-
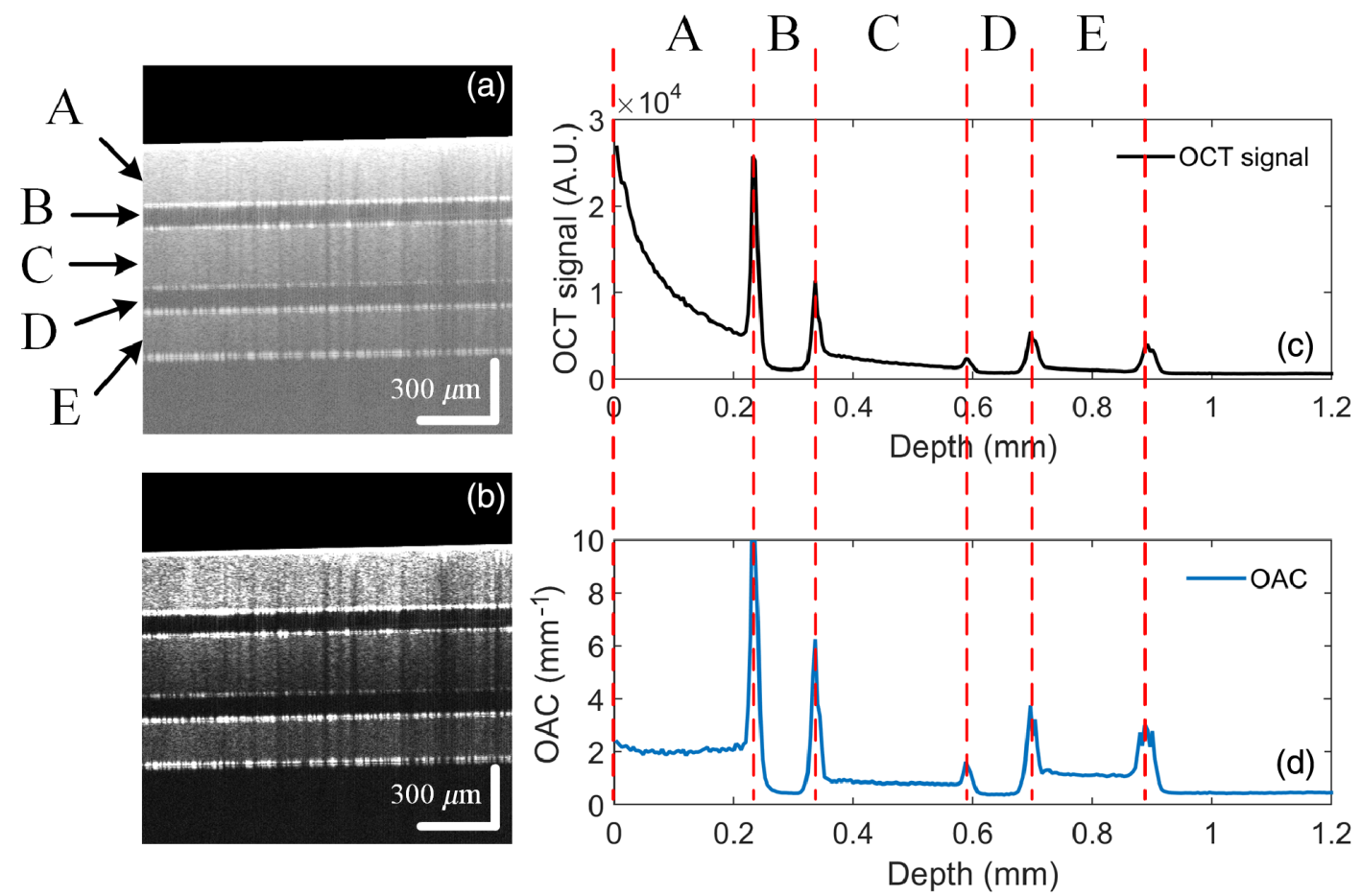

Fig. 8 Estimated OCA for the multilayer phantom by ODRE. (a) OCT intensity image; A, C, and E are $15 \%, 5 \%$, and $10 \%$ of phantom, respectively. $B$ and $D$ are the coverslips. (b) Corresponding OAC image obtained by ODRE. (c) A typical A-scan within (a); (d) corresponding OAC of (c). 


\section{Baseline en face OCT angiogram}
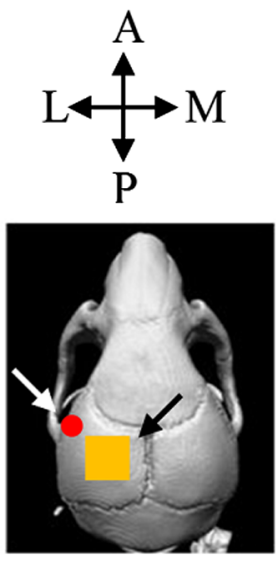

(a)
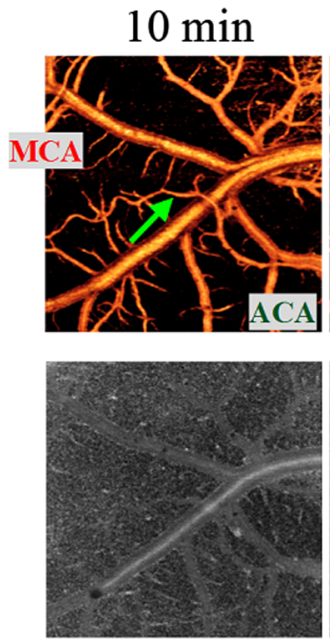

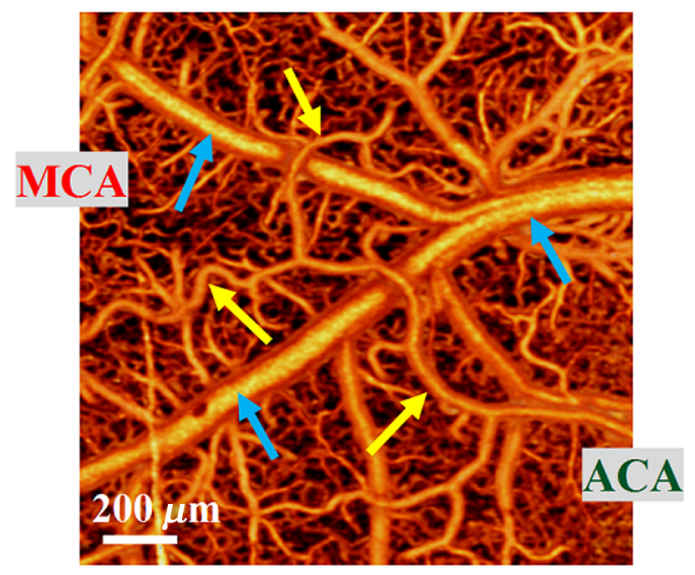

(b)

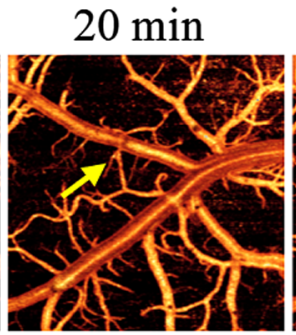

$30 \mathrm{~min}$
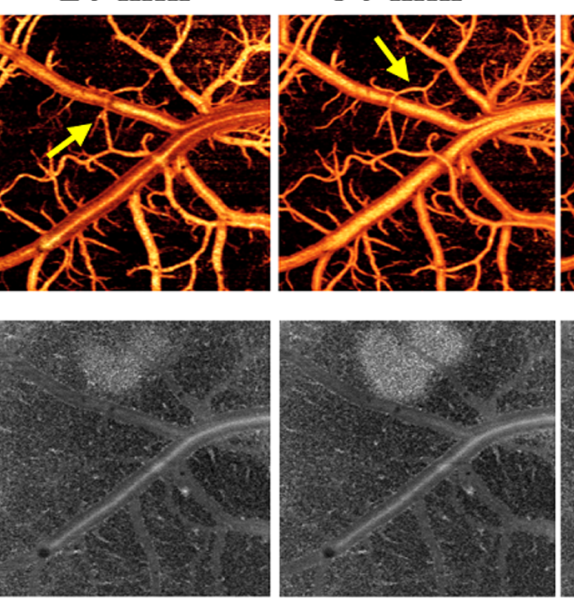

\section{Baseline en face OAC image by ODRE}

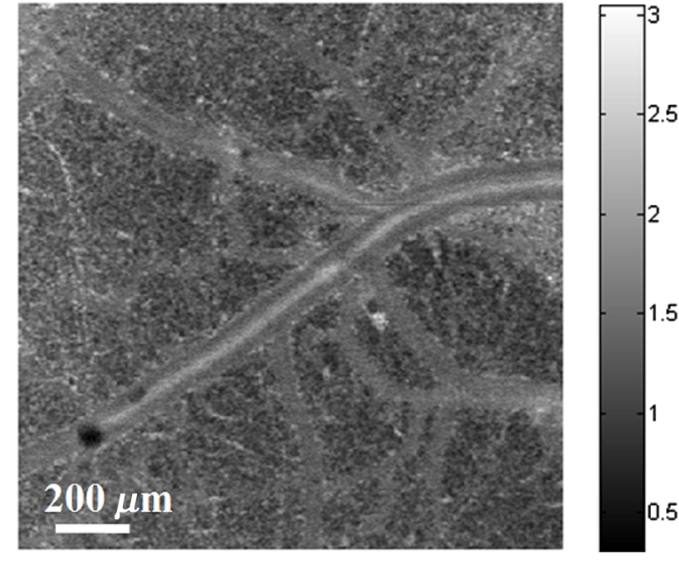

(c)
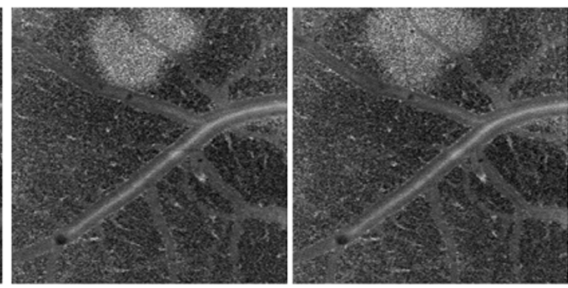

(d)
$40 \mathrm{~min}$

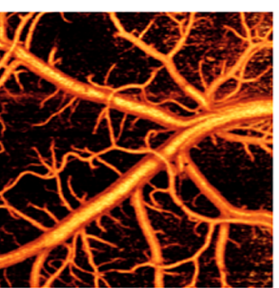

$60 \mathrm{~min}$

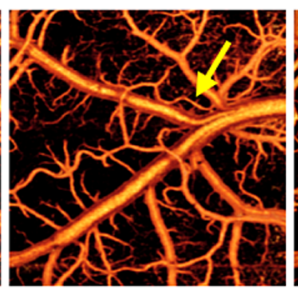

$120 \mathrm{~min}$
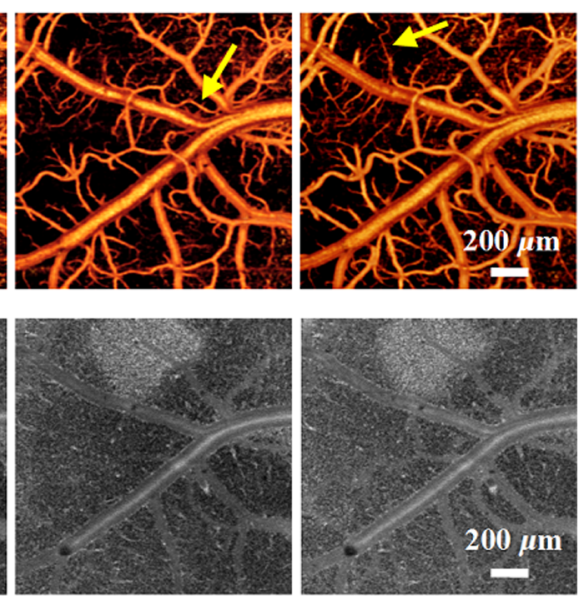

Fig. 9 (a) Scan of a subject's skull; the ROI for data acquisition is indicated by a black arrow; the red dot indicated by the white arrow is the position of the ET-1 injection. (b) The baseline en face OCT angiogram, up to $150-\mu \mathrm{m}$ depth. (c) Average en face OAC plots with a depth range of 151 to $300 \mu \mathrm{m}$, with the OACs in the range of 0.3 to $3\left(\mathrm{~mm}^{-1}\right)$. (d) The results of OCT angiograms and OAC images of the mouse cortex during ET-1-induced MCAO, respectively; the white bar represents $200 \mu \mathrm{m}$.

induced middle cerebral artery occlusion (MCAO) model. ${ }^{12}$ The stereotaxic coordinates of the ET-1 injection were $-0.83-\mathrm{mm}$ anterior, $-3.36-\mathrm{mm}$ lateral, and $1.0-\mathrm{mm}$ ventral relative to bregma. A dose of $3-\mu \mathrm{L} \mathrm{ET}-1$ (dilute to $100 \mathrm{pmol} / \mathrm{L}$ in $0.9 \%$ normal saline) was injected at $1 \mu \mathrm{L} / \mathrm{min}$. After injection, we waited for $3 \mathrm{~min}$ to ensure that the drug was completely absorbed. The needle was slowly removed, and the mouse was immediately placed under the sample arm to begin the temporal scans. During date acquisition, the mouse was under gas anesthesia (isoflurane) instead of IP anesthesia.

In mouse brain imaging, all the datasets were taken at the depth of $1 \mathrm{~mm}$ to avoid the noise floor. The number of pixels used for exponential fitting is $120(0.3 \mathrm{~mm})$. In Fig. 9(a), the orange box represents the ROI in our experiment, highlighted with a black arrow. As shown in Fig. 9(b), the baseline en face OCT angiogram, up to the depth of $150 \mu \mathrm{m}$, was obtained from the ROI, and yellow arrows indicate arteries whereas blue indicate veins. Figure 9(c) shows the en face OAC image obtained from the same ROI by the ODRE method. The attenuation coefficient of the ischemic region, but not the healthy region, increases with the development of ischemia so that they can be distinguished during the process. The en face OAC images were reconstructed by averaging OACs at a specific depth range (151 to $300 \mu \mathrm{m}$ ) because there is a better contrast between the damaged and the healthy tissues at this range.

The OCT angiography provides the detailed information on cerebral blood flow, whereas the OAC mapping provides information on the optical properties of the tissue. These two techniques can be combined into an indicator to show the status of brain tissue. When the brain tissue is damaged by ischemia, blood perfusion changed first, and OAC changed after a period of time (20 to $40 \mathrm{~min}$ ). Figure 9 (d) shows the changes in cerebral perfusion and OAC of the cerebral cortex in mice with focal cerebral ischemia over time. 
As shown in Fig. 9(d), when ET-1 was injected, due to a high concentration of ET-1, the arteries and most capillaries near the injection point (a red dot) were blocked rapidly and the blood supply reached a minimum level within $10 \mathrm{~min}$. However, the arteries far from the injection site were not obstructed, which may be due to some compensatory mechanisms, such as anastomosis of MCA and ACA. ${ }^{24}$ The veins also remained unchanged. Part of the reason might be that their vascular walls are relatively thin and the walls are almost unaffected by ET-1. During the period from 20 to $120 \mathrm{~min}$, arteries recovered gradually, as indicated by the yellow arrows in Fig. 9(d), with part of the capillaries also returning to normal.

As shown in Fig. 9(d), the white area gradually appeared in the OAC images over time, indicating that the backscattering of light increased and reflected that the optical properties of the tissue have changed. The white region was located near the blocked artery, suggesting that tissue in this area has been infarcted due to insufficient supply of blood and oxygen.

In the ischemic region, lesions did not occur immediately at the onset of occlusion, and the lesion appeared and the size of the lesion area increased over time. In the later stage, after the effect induced by ET-1 disappeared, blood vessels began to recover gradually. However, we can see that the injured brain cells have not recovered, which indicates that the damage to the brain cells is irreversible. This result is consistent with the conclusion of the reference. ${ }^{9}$

To investigate the effects of cerebral ischemia on OAC in more detail, we studied the OAC of brain tissue in damaged and undamaged areas. The results are shown in Fig. 10.

Figure 10(a) shows a 40-min OCT angiogram, and Fig. 10(b) shows a corresponding time OAC image. The white line in Fig. 10(a) and the yellow line in Fig. 10(b) represent the same cross-sectional location that passes through the damaged cerebral region. The OCT structural images [Figs. 10(c)-10(e)], OCT angiograms [Figs. 10(f)-10(h)], and OAC images [Figs. 10(i)-10(k)] all are at this cross-sectional location.

From the OCT structural images, we can see that the penetration depth of the OCT light (green box) at baseline is significantly greater than that at 40 and $120 \mathrm{~min}$. Penetration depth refers to the depth at which the signal intensity is reduced to $5 \%$ of its initial value. The penetration depth is $>1 \mathrm{~mm}$ at baseline and $<0.5 \mathrm{~mm}$ at 40 and $120 \mathrm{~min}$. This indicates that the attenuation of light in the damaged cerebral tissue is enhanced. In the OCT angiograms, the blood vessels are abundant at baseline, and they are minimal at $40 \mathrm{~min}$ and recover at $120 \mathrm{~min}$. It is not easy to identify changes in OAC in the damaged region from the cross-sectional images, so we chose a specific depth range of 151 to $300 \mu \mathrm{m}$ (yellow box) and plotted the average OAC curve in that range. Figures 10(1)-10(n) are the average OAC curves for the baseline, $40 \mathrm{~min}$, and $120 \mathrm{~min}$, respectively. From these curves, it is noticeable that the average OACs across the 151- to $300-\mu \mathrm{m}$ depth of the cross-section are approximately the same at the baseline. In contrast, at 40 and 120 min after ischemia, the $\mathrm{OACs}$ in the middle part (injured region) are significantly higher than those on both sides of the image.

The red and blue curves in Fig. 10(o), respectively, correspond to the damaged region (red region 1) and the nondamaged region (blue region 2) shown in Fig. 10(b). The OAC curve for region 1 significantly increases after ischemia, peaks at $40 \mathrm{~min}$, and then fluctuates slightly. The curve for region 2 does not change noticeably before $50 \mathrm{~min}$, after which the OAC gradually increases and the gap between it and the red curve narrows. This indicates that the brain tissue in region 2 was also affected by ischemia, although it was located relatively far from the ET-1 injection site. Figures $10(\mathrm{p})$ and $10(\mathrm{q})$ are a series of OAC curves for a typical A-scan of region 1 and region 2, respectively, all of which vary with depth. Different colors and shapes are intended to represent different times. The above results show that OAC can be used as an indicator for brain injury.

\section{Discussion}

The method of calculating OAC from OCT signals can be used for in vivo characterization of various tissue types with pathological conditions, such as atherosclerosis, burn scar, glaucoma, ${ }^{25}$ and ischemic brain. ${ }^{9,10}$ Vermeer et al. have confirmed that the DRE method is capable of estimating the OAC in uniform and layered, heterogeneous phantoms. However, the DRE method produces two types of errors. The first type of error increases with depth, mainly because the DRE model uses a finite depth $N$ instead of infinity. This error has no significant effect on the shallow layer when the optical signal is completely attenuated and the SNR is high enough. Noise floor exists in the OCT signal, but the DRE method uses the full-depth OCT signal as an input to estimate the OAC. As a result, a large amount of noise is added to the denominator of Eq. (5), which results in a second error: the shallow $\mathrm{OAC}$ is underestimated. Therefore, for the DRE method, the result of the shallow layer is smaller than it should be, and the result of the deep layer is larger than the value it should have. Cutting signals with a low SNR will shorten the estimated depth of OAC and will increase the error of the first type (see Fig. 5). The smaller the depth range, the sooner the error occurs. The existence of these two errors greatly affects the accuracy of the DRE method and limits its application.

The ODRE method has solved these two errors at the same time. First, we improved the estimation model by compensating the summation of $N$ to infinity by fitting the final end of the OCT signal, which solves the first error. In addition, it is important to note that the ODRE method does not require complete attenuation of the light, so OAC can be estimated in any depth range. Therefore, we can eliminate signals with low SNR and solve the second type of error problem. These advantages make ODRE an attractive alternative for OAC calculation and may be suitable for a wider range of applications.

To verify the performance of ODRE, numerical simulations and phantom experiments were performed, both of which confirmed that ODRE is capable of estimating the OAC from the OCT signals with high accuracy. In the multilayer phantom experiment, we found that for the same concentration of the phantom, the results of the multilayer phantom are less than that of the uniform phantom. It may be that the SNR is lower at a deeper depth. SD-OCT is characterized by a rapid fall-off in sensitivity along depth due to insufficient spectral resolution. Moreover, the aliasing artifact may appear at the maximum imaging depth. It is because the sampling frequency of the signal is close to or even lower than the Nyquist sampling rate at the maximum imaging depth. Increasing the upsampling ratio would decrease the amount of aliasing. ${ }^{26}$

The coherent light attenuation transmitted through turbid media is attenuated due to scattering and absorption. Most OCT systems work in near-infrared regions; they use the optical window from 800 to $1400 \mathrm{~nm}$ in tissue. Hence, the largest part of the OAC might be caused by scattering. ${ }^{27,28}$ In addition, because the OCT system can only receive backscattered light, the absorption coefficient cannot be detected. As a result, the 


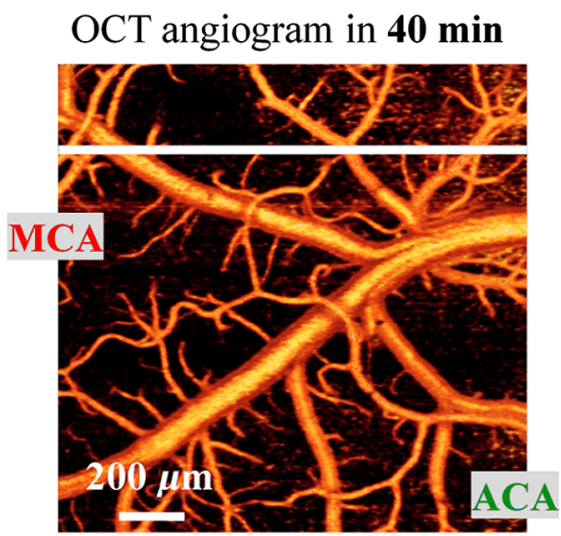

(a)

OAC image in $\mathbf{4 0} \mathbf{~ m i n}$

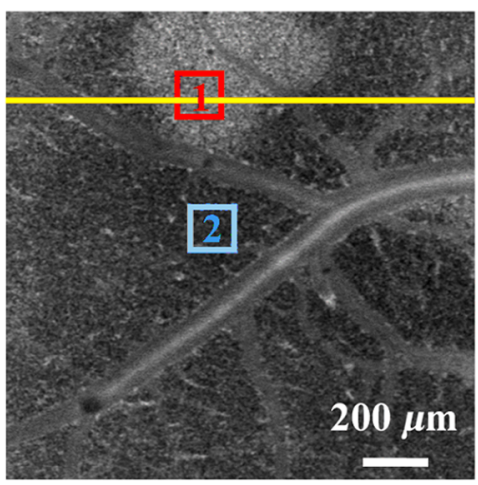

(b)
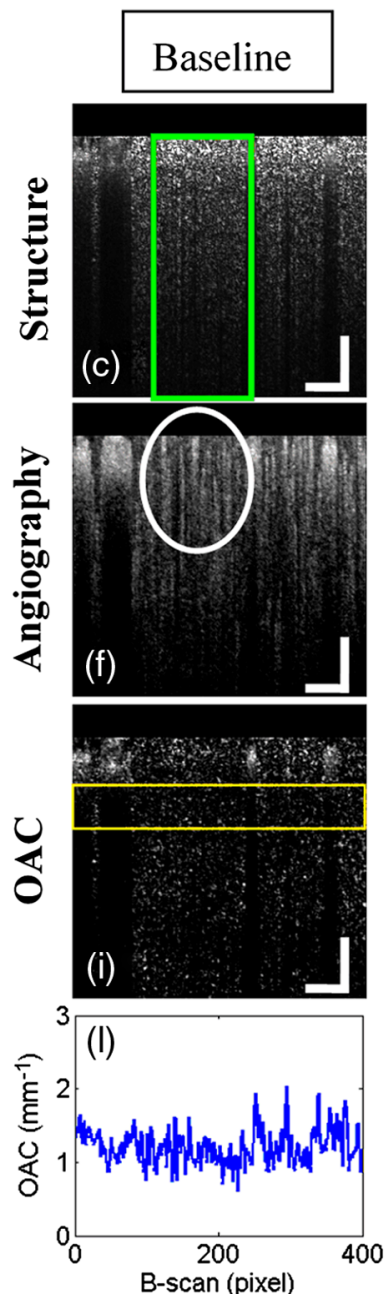
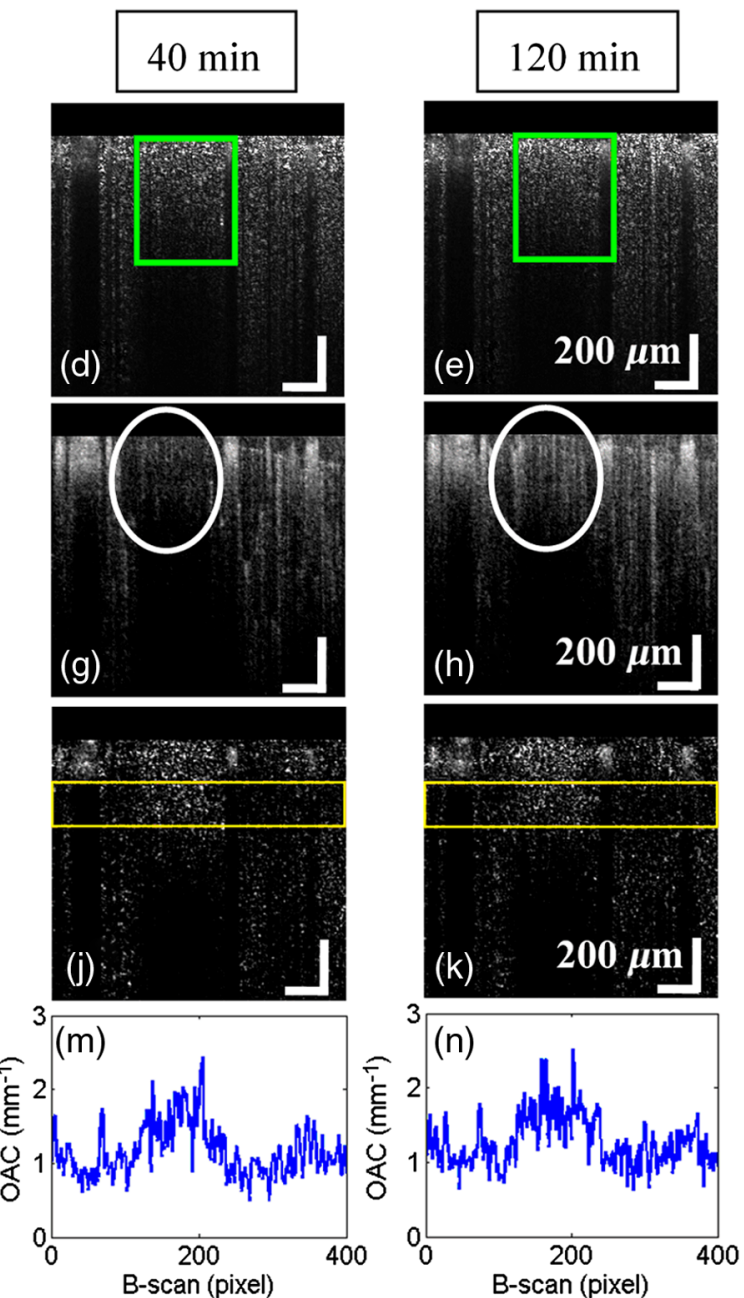

Region size: $10 \times 10$ pixel

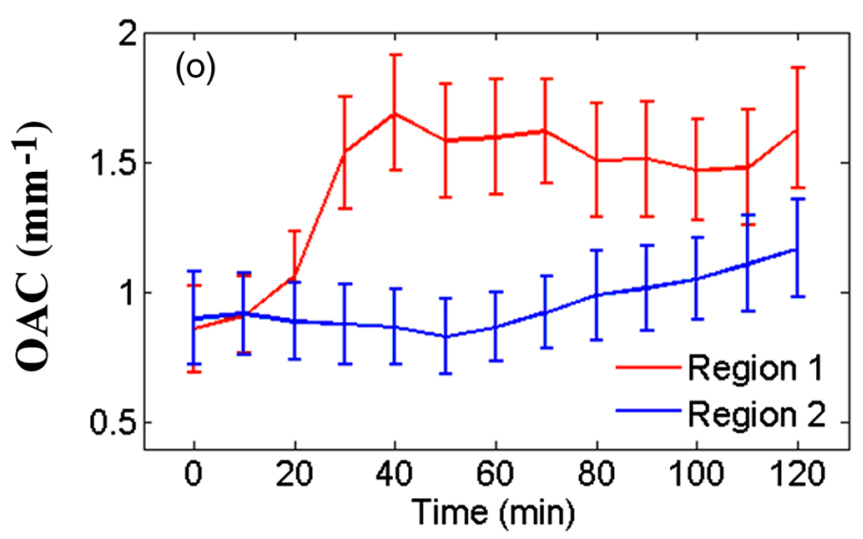

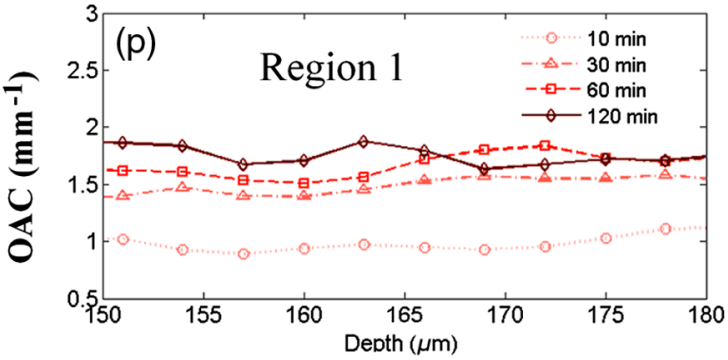

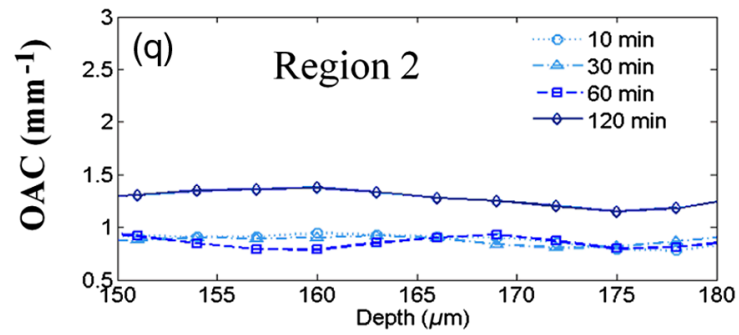

Fig. 10 (a) OCT angiogram at 40 min with the MCA end on the left and the ACA end on the right; the white lines represent the cross sections of the tissue for the OCT structure images (c)-(e) and the OCT angiograms (f)-(h). (b) OAC image at $40 \mathrm{~min}$; the yellow line indicates the cross sections of the tissue for the OAC images (i)-(k) and average OAC curve (l)-(n); the red box 1 and the blue box 2 are the sampling locations chosen representatively as the injured area and the noninjured area, respectively; both region sizes are $10 \times 10$ pixels. (o) The time-varying curve of the OAC in regions 1 and 2 , with all values reported as the mean \pm S. E. M. (p) and (q) OAC curves varying with depth in regions 1 and 2, respectively. 
DRE method assumes that the attenuation is caused by scattering, that is, the backscattered light is a fixed fraction of the attenuated light. However, blood has a strong infrared light absorption characteristic. Ignoring the absorption coefficient will result in the resulting OAC being underestimated.

There are two forms of cell death in the ischemic area: apoptosis and necrosis. Researchers have proposed some convincing conclusions on the reasons for the changes in optical properties. Plesnila et al. ${ }^{29}$ claimed that a mitochondrial protein, apoptosisinducing factor (AIF), plays a role in programmed cell death after focal cerebral ischemia. They found that AIF is localized only in the cytoplasm of almost all striatum and cortical cells 45 min after MCAO. About $1 \mathrm{~h}$ after MCAO, individual cortical cells showed significant AIF staining.

Van der Meer et al. ${ }^{16}$ used OCT to image porcine carotid arteries in vitro and observed an increase in backscattered light in the medial layer after balloon dilation. They hypothesized that the changes in OCT signal could be attributed to this characteristic form of cell death. They then examined the optical properties of granular human fibroblasts in which necrosis or apoptosis was induced. The OAC in necrotic cells decreased from $2.2 \pm$ $0.3 \mathrm{~mm}^{-1}$ to $1.3 \pm 0.6 \mathrm{~mm}^{-1}$, whereas in apoptotic cells, an increase to $6.4 \pm 1.7 \mathrm{~mm}^{-1}$ was observed. ${ }^{30}$ This study indicates that, in our experiment, the white region with increased OAC, as shown in Fig. 9(d), may be the result of apoptosis. This is our preliminary conclusion and we need to conduct follow-up confirmation studies. From the experiments, we observed that OAC mapping can distinguish the boundaries between infarcts and salvageable tissues at different stages of stroke development. After $2 \mathrm{~h}$ of real-time monitoring of mice with focal cerebral ischemia, it can be seen that when the blood flow was at a minimum, apoptosis of cerebral cortical cells did not occur, but it gradually occurred after reperfusion. It is possible that reperfusion aggravates brain damage or that apoptosis occurs only after a period of ischemia. The OAC of nondamaged area also increased over time, which explained why the contrast of the OAC image in Fig. 9(d) (120 min) is lower than before. At the same time, further studies are needed to correlate the local contrast changes with the histological results and explore the relationship between brain damage and OAC.

In conclusion, OCT can provide high-resolution microvascular and structural images of the rodent brains in vivo. The OAC mapping estimated from the OCT signals can help in depicting the pathological changes in brain tissue and may serve as an indicator of tissue injury, which helps us to improve our understanding of human cerebral diseases.

\section{Disclosure}

The authors declare that there are no conflicts of interest related to this article.

\section{Acknowledgments}

This work was supported in part by the National Natural Science Foundation of China (61771119 and 61601106), the Hebei Provincial Natural Science Foundation of China (H2018501087 and H2018501067), and Fundamental Research Funds for the Central Universities (N172304034 and N172304029).

\section{References}

1. P. H. Tomlins and R. K. Wang, "Theory, developments and applications of optical coherence tomography," J. Phys. D 38(15), 2519-2535 (2005).
2. F. L. Lizzi et al., "Relationship of ultrasonic spectral parameters to features of tissue microstructure," IEEE Trans. Ultrason. Ferroelectr. Freq. Control 34(3), 319-329 (1987).

3. M. Judith and B. Irving, "Elastic-scattering spectroscopy and diffuse reflectance," in Biomedical Photonics Handbook, CRC Press, Boca Raton (2003).

4. S. Kawauchi et al., "Light scattering change precedes loss of cerebral adenosine triphosphate in a rat global ischemic brain model," Neurosci. Lett. 459(3), 152-156 (2009).

5. D. J. Faber, M. C. Aalders, and T. G. van Leeuwen, "Curve fitting for quantitative measurement of attenuation coefficients from OCT images," Proc. SPIE 5690, 325-334 (2005).

6. P. P. Dan et al., "Signal attenuation and box-counting fractal analysis of optical coherence tomography images of arterial tissue," Biomed. Opt. Express 1(1), 268-277 (2010).

7. M. Almasian et al., "Validation of quantitative attenuation and backscattering coefficient measurements by optical coherence tomography in the concentration-dependent and multiple scattering regime," J. Biomed. Opt. 20(12), 121314 (2015).

8. K. A. Vermeer et al., "Depth-resolved model-based reconstruction of attenuation coefficients in optical coherence tomography," Biomed. Opt. Express 5(1), 322-337 (2014).

9. U. Baran, Y. Li, and R. K. Wang, "In vivo tissue injury mapping using optical coherence tomography based methods," Appl. Opt. 54(21), 6448-6453 (2015).

10. U. Baran et al., "Automated segmentation and enhancement of optical coherence tomography-acquired images of rodent brain," J. Neurosci. Methods 270, 132-137 (2016).

11. Z. Ma et al., "In vivo assessment of wall strain in embryonic chick heart by spectral domain optical coherence tomography," Appl. Opt. 54(31), 9253-9257 (2015).

12. J. Liu et al., "Hemodynamic changes in a rat parietal cortex after endothelin-1-induced middle cerebral artery occlusion monitored by optical coherence tomography," J. Biomed. Opt. 21(7), 075014 (2016).

13. Y. Watanabe, "Real time processing of Fourier domain optical coherence tomography with fixed-pattern noise removal by partial median subtraction using a graphics processing unit," J. Biomed. Opt. 17(5), 050503 (2012).

14. S. Moon, S. W. Lee, and Z. Chen, "Reference spectrum extraction and fixed-pattern noise removal in optical coherence tomography," Opt. Express 18(24), 24395-24404 (2010).

15. T. G. Van Leeuwen, D. J. Faber, and M. C. Aalders, "Measurement of the axial point spread function in scattering media using single-mode fiber-based optical coherence tomography," IEEE J. Sel. Topics Quantum Electron. 9(2), 227-233 (2003).

16. F. J. van der Meer et al., "Quantitative optical coherence tomography of arterial wall components," Laser Med. Sci. 20(1), 45-51 (2005).

17. G. T. Smith et al., "Automated, depth-resolved estimation of the attenuation coefficient from optical coherence tomography data," IEEE Trans. Med. Imaging 34(12), 2592-2602 (2015).

18. S. H. Yun et al., "High-speed spectral-domain optical coherence tomography at $1.3 \mu \mathrm{m}$ wavelength," Opt. Express 11(26), 3598-3604 (2003).

19. G. Liu et al., "A comparison of Doppler optical coherence tomography methods," Biomed. Opt. Express 3(10), 2669-2680 (2012).

20. G. Gong, H. Zhang, and M. Yao, "Speckle noise reduction algorithm with total variation regularization in optical coherence tomography," Opt. Express 23(19), 24699-24712 (2015).

21. A. Pižurica et al., "Multiresolution denoising for optical coherence tomography: a review and evaluation," Curr. Med. Imaging Rev. 4(4), 270-284 (2008)

22. B. Karamata et al., "Speckle statistics in optical coherence tomography," J. Opt. Soc. Am. A 22(4), 593-596 (2005).

23. M. Pircher et al., "Speckle reduction in optical coherence tomography by frequency compounding," J. Biomed. Opt. 8(3), 565-569 (2003).

24. U. Baran, Y. Li, and R. K. Wang, "Vasodynamics of pial and penetrating arterioles in relation to arteriolo-arteriolar anastomosis after focal stroke," Neurophotonics 2(2), 025006 (2015).

25. D. S. J. Van et al., "The effect of glaucoma on the optical attenuation coefficient of the retinal nerve fiber layer in spectral domain optical coherence tomography images," Invest. Ophthalmol. Visual Sci. 53(4), 2424-2430 (2012). 
Liu et al.: Optimized depth-resolved estimation to measure optical attenuation coefficients from optical coherence...

26. K. K. H. Chan and S. Tang, "High-speed spectral domain optical coherence tomography using non-uniform fast Fourier transform," Biomed. Opt. Express 1(5), 1309-1319 (2010).

27. S. J. Matcher, M. Cope, and D. T. Delpy, "In vivo measurements of the wavelength dependence of tissue-scattering coefficients between 760 and $900 \mathrm{~nm}$ measured with time-resolved spectroscopy," Appl. Opt. 36(1), 386-396 (1997).

28. M. Hohmann et al., "Extension of depth-resolved reconstruction of attenuation coefficients in optical coherence tomography for slim samples," Proc. SPIE 9792, 97920P (2015).

29. N. Plesnila et al., "Nuclear translocation of apoptosis-inducing factor after focal cerebral ischemia," J. Cerebr. Blood Flow Metab. 24(4), 458-466 (2004).
30. F. J. van der Meer et al., "Apoptosis- and necrosis-induced changes in light attenuation measured by optical coherence tomography," Laser Med. Sci. 25(2), 259-267 (2010).

Zhenhe $\mathrm{Ma}$ is an associate professor and a master student mentor at the School of Control Engineering, Northeastern University, Qinhuangdao, China. He was a postdoctoral fellow at the Oregon Health \& Science University (2009-2010). His main research directions are optical coherence tomography, infrared spectroscopy, and Raman spectroscopy.

Biographies of the other authors are not available. 